\title{
Unique Radio Pulsar Geminga
}

\author{
V.M. Malofeev and O.I. Malov \\ Pushchino Radio Astronomy Observatory, Lebedev Physical Institute, \\ 142292, Pushchino, Moskow reg., Russia
}

\begin{abstract}
This pulsar has unique properties of radio emission, the most steep spectrum, the largest changes of pulse widths and phases and the presence of giant pulses.
\end{abstract}

We are continuing Geminga observations and present some new data. The measurements were performed with the Large Phased Array at $102 \mathrm{MHz}$ and cross-type Radio Telescope at lower frequencies (Pushchino). The examples of mean profiles at 87, 59 and $39 \mathrm{MHz}$ are shown at Fig.1. We present evidence of the frequency dispersion of Geminga integrated pulses at $40.7 \mathrm{MHz}$ with $D M \approx 2.9 \mathrm{pc} \cdot \mathrm{cm}^{-3}$ (Fig.1). All observations showed the temporal changes of the form, width and the pulse phase (Malofeev \& Malov 1997, 2000 a, b). This phenomenon can be explained by the presence a few giant pulses during $\sim 0.1 \%$ of observing time, when the flux density can be $10-20 \mathrm{Jy}$ (Fig.2).

Geminga showed the steepest spectrum not only among pulsars, but among all radio sources. The estimated spectral index is greater than 5. Spectrum has sharp low-frequency turnover with a maximum about $60 \mathrm{MHz}$. The data from the lowest frequencies is from Ul'yanov (private communication) at $25 \mathrm{MHz}$; Ramachandran et al. 1998 at 35 and $318 \mathrm{MHz}$; our measurements at 39, 59, 87 and $102 \mathrm{MHz}$; Xilouris (privat comm.) at $47 \mathrm{MHz}$; Lazio, Kassim, 2000 at 74 $\mathrm{MHz}$; Kuzmin et al. 1997; Shitov et al. 1997, Vats et al. 1999 at $103 \mathrm{MHz}$; Burderi et al. 1999 at 317, 410 and $1400 \mathrm{MHz}$.

The absence of pulsar signal at few frequencies (47 and $74 \mathrm{MHz}$ ) can be explained by a short-time observations during a few hours, take into account the strong changes of Geminga's flux density from day to day (Malofeev \& Malov 1997).

Acknowledgments. This work was partly supported by RFBR and INTAS (grant No 96-0154).

\section{References}

Malofeev V.M., Malov O.I. 1997, Nature, 389, 697.

Malofeev V.M., Malov O.I. 2000a, Astron. Rep., in press.

Malofeev V.M., Malov O.I. 2000b, Pulsar Astronomy -2000 and Beyond. ASP Conference Series (M.Kramer, N.Wex and R.Wielebinski eds.), in press. 

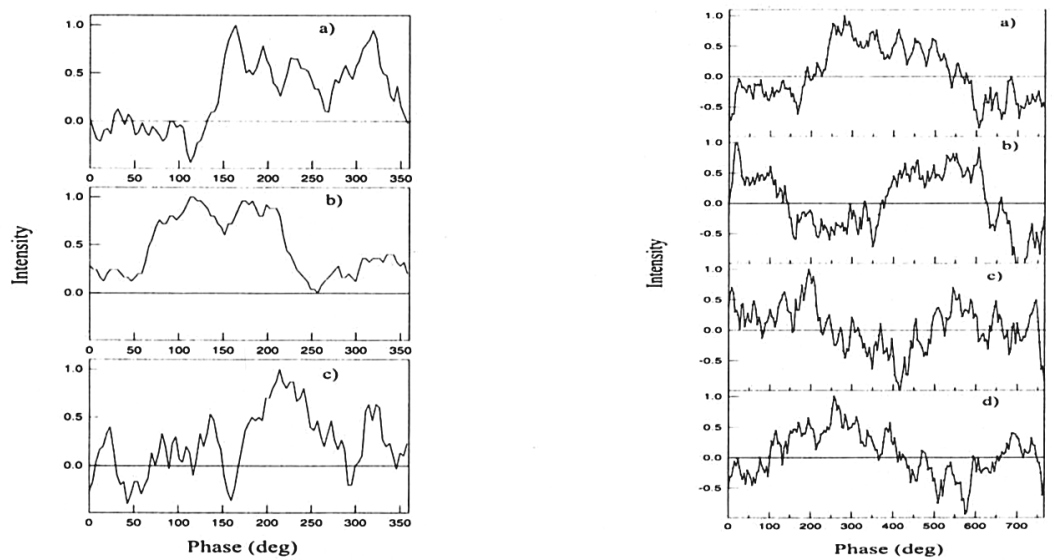

Figure 1. Left panel: Examples of mean pulse profiles at three frequencies: a) $87 \mathrm{MHz}, 2744$ individual pulses, 27.01.98; b) $58.7 \mathrm{MHz}$, 9472 pulses, 5 days; c) $39.05 \mathrm{MHz}, 9920$ pulses, 4 days. Right panel: Examples of mean pulse profiles dispersion at 40.977 - a); 40.817 b); 40.657 - c) and $40.497 \mathrm{MHz}$ - d). Sum of 318 individual pulses (21.08.97).
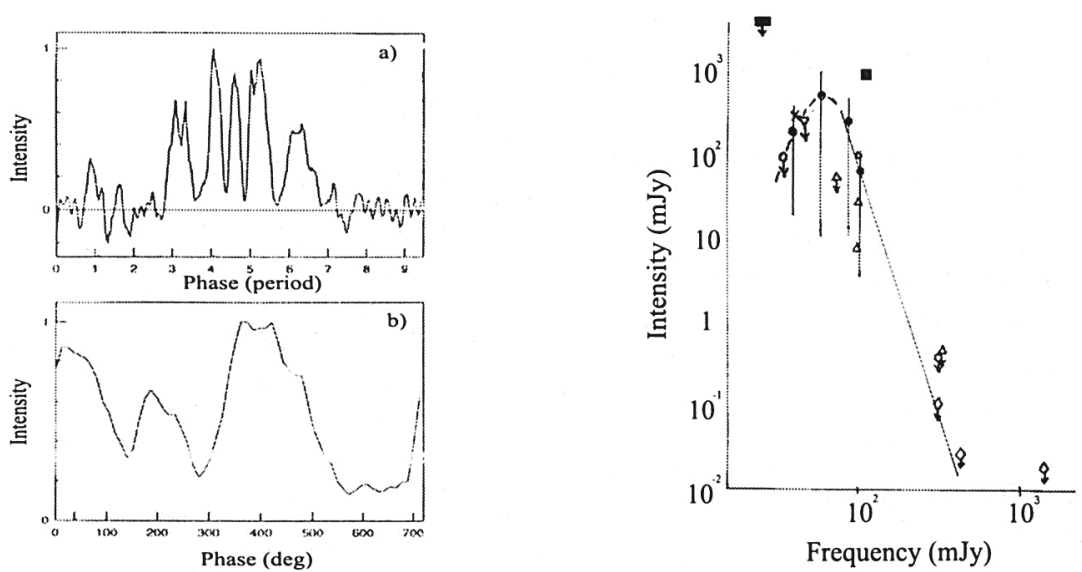

Figure 2. Left panel: Examples of group of very strong pulses at $87 \mathrm{MHz}$ (12.05.98): a) 10 pulsar periods; b) 2 periods. Right panel: Compiled spectrum of Geminga. 